Pasterova 2, 11000 Beograd

Serbia and Montenegro

Correspondence: S Milenković

Tel: + 38111688997

Fax: + 381112688164

E-mail: milenkov@net.yu

Eye (2005) 19, 917-920. doi:10.1038/sj.eye.6701670; published online 10 September 2004

Sir,

\section{Deep lamellar keratoplasty in corneal dermoid}

Corneal dermoids are a rare cause of corneal opacification consisting of abnormal mesoblastic tissue covered by the epithelium. They can involve the deeper layers of cornea leaving an intact descemet's membrane and endothelium. Occasionally, anterior segment structures may be involved by the choristomatous growth and there may occur accompanying ocular malformations. A surgical intervention in the form of lamellar keratoplasty (LK) or penetrating keratoplasty (PK) is warranted on accounts of cosmesis, discomfort, and primarily because they are located in the visual axis. Recently, we treated a child with a corneal dermoid encroaching on to the visual axis. An anterior deep lamellar keratoplasty was performed after the assessment of depth of involvement of the corneal mass by ultrasound biomicroscopy. To our knowledge this is the first report of a successful deep LK in a patient with a corneal dermoid.

\section{Case report}

An 8-year-old boy presented with the complaints of pinkish mass and decreased vision in his left eye. This mass was present since birth and had gradually increased in size. The child was the product of normal uncomplicated gestation and had normal development.

Ocular examination disclosed a normal right eye with a visual acuity of $6 / 6$. Left-eye visual acuity was hand movements close to face. Constant divergent squint of approximately 30-prism diopter was present in the left eye. Eyelids were normal in both eyes. Left eye showed a vascularized, moderately elevated, sharply

circumscribed pinkish mass $6 \times 6 \mathrm{~mm}^{2}$ in size overlying the cornea superonasally. Also present was a ring of lipid deposit around the mass in the adjacent clear cornea (Figure 1a). The remaining cornea was clear. Anterior segment was otherwise normal. Clinically, a diagnosis of corneal dermoid was made. Ultrasound biomicroscopy
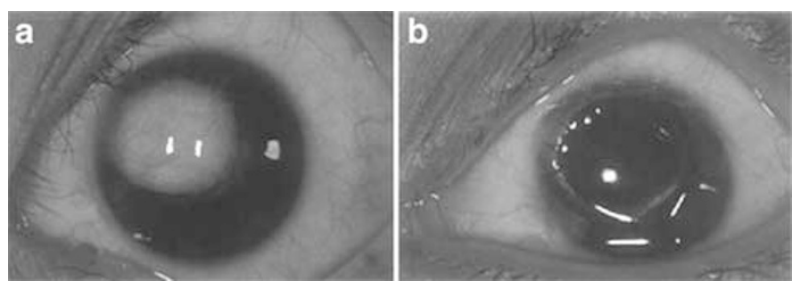

Figure 1 (a) Clinical photograph of left eye showing smooth, elevated, sharply circumscribed mass with an arc of lipid in adjacent cornea. (b) Left eye 6 months after DLK showing clear graft.
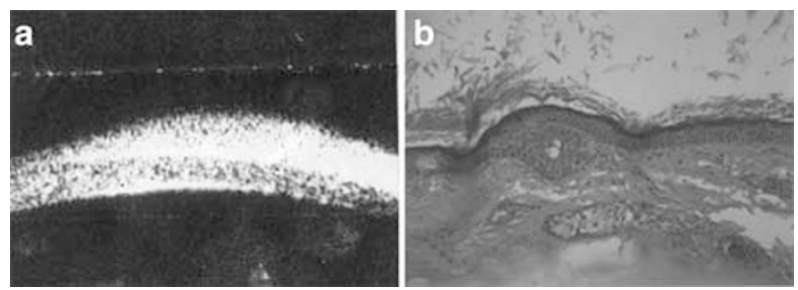

Figure 2 (a) Ultrasound biomicroscopic picture showing highly echogenic lesion occupying the superficial $60 \%$ of the cornea. (b) Histopathology slide showing thick keratinized epithelium and a sebaceous gland enmeshed in connective tissue $(\mathrm{H} \& \mathrm{E}, \times 25)$.

(UBM) disclosed a highly echogenic lesion occupying the superficial $60 \%$ of the cornea. Deeper part of stroma, descemet's membrane and endothelium were not involved (Figure 2a). An anterior deep LK (DLK) was planned.

After a partial thickness corneal trephination with $6 \mathrm{~mm}$ trephine, a superficial keratectomy involving the mass was performed. A disposable 30-gauge needle was inserted deeply with bevel down into the paracentral stroma and air was injected. A small opening was made in the air bubble and the remaining stromal layers were removed till the descemet's membrane was bared of the stroma. Donor corneal button $6.5 \mathrm{~mm}$ sized, stripped of descemet's membrane was sutured onto the bare descemet's membrane. In all, 16 interrupted 10/0 nylon sutures were given. Postoperatively, the eye was treated with topical prednisolone acetate $(1 \%)$, tobramycin $(0.3 \%)$ and a tear substitute four times a day. Histopathology of the corneal dermoid revealed thick keratinized epithelium and sebaceous glands enmeshed in connective tissue (Figure 2b). Patient had an uneventful postoperative course. Visual acuity improved to 3/60 and is currently receiving ambylopia therapy. At 6 months postoperatively the graft has remained transparent with no interface scarring (Figure 1b). 


\section{Comment}

Corneal dermoid is a congenital benign tumour consisting of tissues of ectodermal and mesodermal origin appearing as raised yellowish white vascularized bulbous lesions. ${ }^{1}$

Mann $^{2}$ classified corneal dermoids into three broad types. Our case belonged to grade II with corneal involvement sparing deep stroma, descemet's membrane, and endothelium. This was elucidated well on UBM.

The surgical management of corneal dermoid depends on the size, site and depth of involvement. ${ }^{3}$ Simple excision is generally not sufficient by itself to manage an extensive lesion. ${ }^{4}$

Corneal dermoids with no anterior chamber involvement require LK in which the lesion is excised to its entirety and a lamellar graft is tailored to fit the defect. ${ }^{1}$

LK has the advantage of avoiding most postoperative complications associated with PK especially less risk of allograft rejection but has the major disadvantage of interface scarring and hazy graft. Of late, DLK is being performed more commonly over LK/PK with minimal reports of interface scarring. We decided to perform DK using big bubble technique as UBM elucidated the lesion to be distinct and sparing the underlying descemet's membrane and endothelium.

The case is being reported because of the rarity of the condition and use of relatively new diagnostic tool UBM to assist in its management. To the best of our knowledge DLK has not been previously reported in corneal dermoid management.

\section{References}

1 Henkind P, Marinoff G, Manas A, Friedman A. Bilateral corneal dermoids. Am J Ophthalmol 1973; 76: 971-977.

2 Mann I. Development Abnormalities of the Eye. Lippincott: Philadelphia, 1957, pp 357-364.

3 Golubovic S, LatKovic Z, Horyatic OM. Surgical treatment of larger corneal dermoid. Doc Ophthalmol 1995; 91: 25-32.

4 Mohan M, Mukherjee G, Panda A. Clinical evaluation and surgical intervention of limbal dermoid. Indian J Ophthalmol 1981; 29: 69-73.

R Arora, V Jain and D Mehta

Cornea Services, Guru Nanak Eye Center

New Delhi 110002, India

Correspondence: R Arora

D-1, Nizammudin

West New Delhi 110013, India

Tel: + 911124351415

E-mail: aroraj@del3.vsnl.net.in
Eye (2005) 19, 920-921. doi:10.1038/sj.eye.6701672;

published online 3 September 2004

Sir,

Stenotrophomonas maltophilia keratitis after penetrating keratoplasty

Stenotrophomonas maltophilia is an aerobic, Gram-negative ubiquitous bacillus, isolated from water, soil, plants, and animals. ${ }^{1}$ Previously described to be of limited pathogenic potential, it is now emerging as an important nosocomial pathogen. ${ }^{2}$ Microbial keratitis due to $S$. maltophilia is rare with only 11 cases reported in literature. ${ }^{3-7}$ We report a case of S. maltophilia keratitis following penetrating keratoplasty that was managed by topical fluoroquinolone monotherapy.

\section{Case report}

A 70-year-old lady presented with diminished vision of 1 week's duration in her right eye. In the affected eye a penetrating keratoplasty was done for a corneal scar (a sequel of burnt-out trachoma) 5.5 months earlier. Postoperatively, a persistent epithelial defect resolved over 2 months with topical preservative-free tear substitutes, antibiotic eyedrops and a bandage contact lens. On examination, her best-corrected visual acuity in the right eye was $20 / 400$. She had lagophthalmos with no corneal exposure. The lid margins were thickened and irregular with significant meibomitis. Trichiasis was not noted. The graft-host junction was well-apposed. There was no bandage contact lens. There was a central epithelial defect with an underlying stromal infiltrate $(3.5 \times 3.7 \mathrm{~mm})$ and surrounding stromal oedema (Figure 1a). The remaining details were not visualised. An ultrasound B scan of the right eye was normal. Grams, Giemsa, and $\mathrm{KOH}$ stains of the corneal scrapings revealed no organisms. She was started on half-hourly fortified Cefazolin eyedrops $(50 \mathrm{mg} / \mathrm{ml})$ and fortified Gentamicin eyedrops $(14 \mathrm{mg} / \mathrm{ml})$. These were discontinued after 2 days due to significant growth of Gram-negative bacilli in culture, sensitive only to ciprofloxacin and chloramphenicol. The organism was identified as Stenotrophomonas maltophilia by API 20NE (API, Biomerieux, France). Gradual resolution with $0.3 \%$ ciprofloxacin hydrochloride eyedrops was noted. A measure of $0.1 \%$ betamethasone sulphate was added to reduce inflammation. The lesion healed after 2.5 months of therapy (Figure 1b). She is currently awaiting a regraft in the right eye. 